\title{
IMPORTANCIA DE LA CONVIVENCIA FAMILIAR PARA LA SALUD DEL ADULTO MAYOR EN LAS CASAS DE RETIRO Y/O ASILO
}

\author{
IMPORTANCE OF FAMILY COEXISTENCE FOR THE HEALTH OF THE ELDERLY \\ ADULT IN RETIREMENT AND / OR ASYLUM HOUSES
}

\author{
Jesús Manuel Guzmán-Vidal ${ }^{1}$ *iD. \\ 1. Escuela Judicial del Estado de Tabasco, México. lic.jmgv78@gmail.com \\ * Autor de correspondencia: Jesús Manuel Guzmán-Vidal, correo electrónico: lic.jmgv78@gmail.com
}

\section{RESUMEN}

Este artículo aborda el estudio de las secuelas y daños que se generan en personas adultas mayores ocasionados por el abandono y falta de convivencia con sus familiares, particularmente el caso de aquellos que se encuentran ingresados en casas de retiro o también llamados asilos. El deterioro emocional y psicológico que padecen por la frágil o nula relación con familiares desencadena una serie de eventos negativos tales como falta de apetito, depresión, aislamiento, negatividad a realizar actividades grupales o las inherentes a su óptima motricidad. Esta serie de eventos, los lleva a presentar patologías que terminan por acabar con su vida a corto plazo. Empero, los eventos negativos antes aludidos, pueden ser anulados a través de la implementación de los círculos restaurativos, en los que las personas adultas mayores cuenten con la oportunidad de expresar sus sentimientos y emociones de manera libre, espontánea y respetuosa, que les propicie una mejor comunicación y relación con familiares. Afianzando con esto, el reforzamiento de los lazos afectivos, el mejoramiento emocional y de salud del adulto mayor.

Palabras clave: adulto mayor; asilo de ancianos; circulo restaurativo.

Cómo citar:

Jerónimo-García, Gloria María. (2021). Importancia de la convivencia familiar para la salud del adulto mayor en las casas de retiro y/o asilo. Revista de Investigaciones Universidad del Quindio, 33(S2), 183-190. https://doi. org/10.33975/riuq.vol33nS2.630 


\begin{abstract}
This article addresses the study of the consequences and damages that are generated in older adults caused by abandonment and lack of coexistence with their relatives, particularly the case of those who are admitted to retirement homes or also called asylums. The emotional and psychological deterioration suffered by the fragile or null relationship with relatives triggers a series of negative events such as lack of appetite, depression, isolation, refusal to carry out group activities or those inherent to their optimal motor skills. This series of events leads them to present pathologies that end up ending their life in the short term. However, the aforementioned negative events can be canceled through the implementation of restorative circles, in which older adults have the opportunity to express their feelings and emotions in a free, spontaneous and respectful way, which encourages them a better communication and relationship with family members. Strengthening with this, the reinforcement of affective ties, the emotional improvement and health of the elderly.
\end{abstract}

Keywords: Elderly; retired people center; restorative circle.

\title{
INTRODUCCIÓN
}

La inquietud de abordar este tema se genera con motivo de una visita a la casa del árbol de la Ciudad de Villahermosa Tabasco, México en la cual fue posible palpar la situación de abandono en la que se encuentran inmersos la gran mayoría de personas adultas mayores ahí residentes. Problemática que desencadena además trastornos en la salud de las personas de la tercera edad, abandono provocan el menoscabo en su integridad física y en sus derechos, de manera especial en su derecho a vivir dignamente.

Se propone dentro del esquema de legalidad una ley local que refuerce los criterios antes expuesto así como la ley federal de proveer los derechos de las personas adultas mayores, con el agregado progresista de la reciprocidad de derechos que parten de ellas mimas y se complementan con el ejercicio de sus familiares, así también se propone de manera generar la incorporación de círculos restaurativos, bajo un programa de vida digna, cuyas finalidades se alcanzan anotar en el presente escrito, lográndose con ello el objetivo de dar una propuesta basada en un enfoque holístico, donde el derecho opte por la convivencia armónica entre la manifestación de valores, reforzadas por la visión de protección por parte del estado a las personas que conforman el grupo vulnerable de adultos mayores.

\section{METODOLOGÍA}

Este artículo tiene como objetivo, resaltar la problemática que se suscita al interior de las casas de retiro y/o asilos, así como las consecuencias de salud y emocional derivada por la actitud de abandono o desinterés desplegadas por los familiares hacia los adultos mayores residentes.

Siendo que después de reflejarse estadísticas y muestreos se buscó la interpretación del derecho a la vida digna y demás derechos que la enarbolan como el derecho a la salud, bienestar, proyecto de vida, entre otros tomando como referencia lo estipulado por el derecho internacional de los derechos humanos, seguidamente se realizó un análisis partiendo de los valores y principios que trae aparejado el derecho plasmado en leyes y que en ocasiones pasan desapercibidos, pero son indispensables rescatarlos y así captar más a fondo la realidad que viven las personas adultas mayores, y de cómo se ejerce la tutela por parte del estado en la intensión de institucionalizar los asilos o casas de retiro. 


\section{RESULTADOS}

\section{Acercamiento a la situación del adulto mayor en México}

De acuerdo con la encuesta especial del Instituto Nacional de Estadística y Geografía del año dos mil quince, en nuestro país residen 15.4 millones de personas de 60 años o más, de las cuales 1.7 millones viven solas. Sólo 41.4\% son económicamente activos, y 69.4\% presentan algún tipo de discapacidad.

Estos datos plasman que una parte importante de nuestra población está conformada por los grupos de la tercera edad, las cuales a saber de todos conforman grupos en situación de vulnerabilidad cuyos cuidados y atención por parte del estado mexicano debe ser mayor en la garantía de sus derechos, principalmente del derecho a la salud en todos sus aspectos y a la vida digna.

No obstante, de acuerdo a las mismas cifras que proporcionó en el mismo año el Directorio Estadístico Nacional de Unidades Económicas (DENUE, 2018) del INEGI en México existen 819 Asilos y otras residencias para el cuidado del anciano, $85 \%$ son del sector privado y $15 \%$ del sector público. Denotando que en nuestro país existen más centros de cuidados para el adulto en manos de particulares que los administrados por el estado de manera directa.

Como es visible a la realidad hoy en día existe una gran necesidad por parte de las familias de que las personas adultas mayores sean internadas en casas de retiro, por la inminente necesidad de asistencia especializada a largo plazo de la que requieren, debido a enfermedades degenerativas, deterioro físico, fragilidad, entre otros, que dificultan el desarrollo de actividades de la vida diaria de manera independiente.

A esta situación se suman los esquemas económicos de la población que opta por servicios públicos en el cuidado de sus familiares al encontrarse limitados de proveerles tales servicios y apelando a la necesidad de que tengan un lugar digno donde se le provea de espacios para su mayor cuidado y atención, sin que tenga que pasar desapercibido que una vez que las personas adultas mayores son ingresadas en estas casas de asistencia, en la mayoría de los casos son olvidadas por sus familiares, generando con esto una depresión constante que empieza a deteriorar su sistema psicoemocional en el cual sus funciones corporales y psicológicas causan estragos en su persona.

\section{El adulto mayor en Tabasco, México.}

Teniendo como referente en nuestro muestreo la situación que arroja la realidad en el estado de Tabasco. En entrevista realizada recientemente al personal de la casa del árbol en la ciudad de Villahermosa, en la cual dichos entrevistados solicitaron se guardara en el anonimato sus nombres, se hizo alusión a los casos antes mencionados. Como primera entrevistada se habló con la trabajadora social encargada de uno de los turnos, la cual hizo referencia sobre las condiciones de salud y emocional en las que se encentran alguna de las personas allí internas, mismos que presentaban falta de interés en realizar las actividades inherentes a su tratamiento psicomotor, el cual sirve para ayudarles a no perder su movilidad corporal, su sensibilidad sensorial y permitirles tener una correcta habilidad de memoria.

Al cuestionar sobre esta falta de interés, la entrevistada en comento hizo alusión a que estas situaciones se presenta regularmente en aquellos que ya no tienen contacto alguno con sus familiares o la relación que existe, se encuentra fracturada por eventos del pasado, lo que les ocasiona un gran daño emocional. 
Otro de los entrevistados fue el psicólogo de dicha institución quien refirió muy acertadamente sobre las consecuencias que tiene la depresión en el adulto mayor y que repercute en la falta de interés por realizar actividades que deberían ayudarle a sentirse bien. Además, enfatizó en que la mayoría de sus pacientes y más específicamente aquéllos que no reciben visita constantemente se hacen un sinnúmero de preguntas como ¿Por qué mi familia no me visita? ¿Les habrá pasado algo? ¿Acaso ya no les importo? ¿Se habrán olvidado de mí? ¿Habré sido un estorbo para ellos?, entre otros. Cuestionamientos cuyas respuestas causan un efecto negativo y devastador en la condición emocional y de salud de esas personas, pues tienen constantemente la incertidumbre del por qué sus familiares ya no le visitan.

El geriatra asignado, relató que un adulto mayor con depresión severa, empieza a tener complicaciones médicas como gastroenteritis, descompensación corporal, hipertensión arterial, circulación sanguínea anormal y muchas otras enfermedades patológicas que acaban por deteriorar al individuo en mención, al grado de ocasionarle la muerte.

Después de recabar los datos arrojados por los operadores encargados del cuidado de las personas adultas mayores en la casa del árbol, consideramos necesario adentrarnos a un análisis dogmático en el impacto de la tutela de derecho a la vida digna desde una concepción holística como ente, en el que se involucra la situación de las personas adultas mayores para poder dar una solución firme que trascienda a cambios mayúsculos del escenario planteado que sienten y viven estas personas en situación de asilo y que generalmente pasa desapercibida por sus familiares y en ocasiones hasta del propio derecho traducido en sistemas legales e institucionales, propuesta que nos lleve desde el ámbito de la persona misma hacia el derecho.

\section{El adulto mayor y atribuciones de sus derechos.}

Dado lo anterior y apelando la concepción del derecho internacional, de los derechos humanos, tenemos que la Corte Interamericana de los Derechos Humanos, se ha pronunciado que; en esencia, el derecho fundamental a la vida comprende, no solo el derecho de todo ser humano de no ser privado de la vida arbitrariamente, sino también el derecho a que no se le impida el acceso a condiciones que le garanticen una existencia digna. Los estados tienen la obligación de garantizar condiciones que se requieren para que no se produzcan violaciones de ese derecho básico y, en particular, el deber de impedir que sus agentes atenten contra él (Corte IDH, 1999).

Este tribunal internacional, ha interpretado de una forma más clara el menoscabo de la falta de atención en la esfera de la vida digna, aterrizando sobre el sentido de la falta de procuración y cuidado por parte del estado a continuar realizando su proyecto de vida, pues como es el caso de este grupo, poco a poco va recayendo en personas que padecen situación de riesgo y que el gobierno tiene para ellos una obligación superior de protección.

Específica el deber del estado de tomar medidas positivas se acentúa precisamente en relación a la protección de la vida de personas vulnerables e indefensas, en situación de riesgo y continúa especificando; "la privación arbitraria de la vida no se limita, pues, al ilícito del homicidio; se extiende igualmente a la privación del derecho de vivir con dignidad. Esta visión conceptualiza el derecho a la vida como perteneciente, al mismo tiempo, al dominio de los derechos civiles y políticos, así como al de los derechos económicos, sociales y culturales, ilustrando así la interrelación e indivisibilidad de todos los derechos humanos" (Corte IDH, 1999). 
En un sentido de interrelación, el derecho a la vida se concibe aparejado de otros derechos que enarbolan la vida digna y que tiene un sentido de disfrute que no deben quedar fuera del goce de su espera jurídica, los cuales deben traducirse en actos de relación con otras personas y de participación en esos ambientes de relaciones humanas. Que o ayuden a seguir construyendo un proyecto de vida que siga cultivando y arropando su existencia humana.

Sin embargo, cuando se notan alejados de esta situación poco a poco se merma a los ojos de la comunidad que lo rodean un sentido de existencia. Pues sin la menor condición siquiera de crear su proyecto de vida, experimenta un estado de padecimiento equivalente a una muerte espiritual; la muerte física que a ésta sigue, en tales circunstancias, es la culminación de la destrucción total del ser humano. Estos agravios hacen víctimas no sólo a quienes los sufren directamente, en su espíritu y en su cuerpo; "la visión que sostenemos corresponde a creencias profundamente arraigadas en las culturas de los pueblos de América Latina, en el sentido de que la muerte definitiva de un ser humano en el orden espiritual sólo se consuma con el olvido" (Corte IDH, 1999).

Para tener y gozar de una vida digna es necesario tener todos los alcances y las condiciones óptimas para el disfrute de este, sin que la escala cronológica de la vida que la persona pueda enfrentar o las condiciones se conviertan en sinónimos de la lejanía de este derecho que le permitan un sano acceso a una existencia de vida garantizada por la salud (OMS, 1946) la alimentación, el vestido, la vivienda y entre otros ${ }^{1}$

La atribución de responsabilidad en temas de salud, alimentación y otros temas de subsistencia, surge a partir del riesgo conocido por el estado y el deber de prevención correspondiente. No todo tipo de actuación grave a la subsistencia sería atribuible al estado, sino aquel que este conocía y puede prevenir, pues viola el derecho a la vida por que no adopta las medidas necesarias y esperadas, dentro del ámbito de sus atribuciones, para prevenir o evitar el riego al derecho a la vida (Parra, 2013).

La vida no se ve condicionada a un modo tradicional de subsistencia donde solo baste, el comer, el vestir, el asesarse y tener un techo donde dormir. Pues la ausencia de la convivencia en ellos por parte de sus familiares, los aísla no solamente del vínculo familiar, sino también poco a poco los desprende de las pautas culturales, que constantemente la aleja del goce de derechos que se construyen en la relación humana, presentando un panorama de discriminación por parte del grupo inmediato al que pertenecen.

Contrario a ello el derecho también apuesta por un entorno de disfrute en un ejercicio armónico a que los familiares reconozcan y establezcan vínculos de convivencia con su familiar en un acto constante de justicia hacia estas personas en las aproximaciones del ocaso de su vida constituye un derecho inalienable recíproco, tanto de los familiares a conservar la memoria y preservar los valores de los cuales fueron formados, como del adulto mayor en cuanto al disfrute que invoque una reivindicación transformadora hacia instituciones que protejan su campo de vida digna enarboladas con los elementos descritos con anterioridad.

Descrito lo anterior, se busca que el derecho también apueste a la valoración de la buena voluntad de los familiares sobre la cercanía y la relación entre las personas adultas mayores concibiéndolo como un paso importante de una vida física a la memoria familiar y colectiva que se le pueda proveer,

1Entiéndase a la salud como el estado de completo bienestar físico, mental y social, y no solamente la ausencia de afecciones o enfermedades. 
así como una forma de sanar lesiones psicoafectivas que se haya vivido en el transcurso de su vida consiguiendo la integración familiar.

Los asilos, en nuestro país, son instituciones de asistencia donde son alojadas algunas personas de edad avanzada, aparentemente no tienen otra finalidad que el agrupamiento, la reclusión y una supervisión médica elemental (Rodríguez, 2000).

Es propio de la naturaleza humana que las personas dentro de los asilos que se encuentran dentro de una situación de abandono y que como consecuencia carece de un contacto afectivo por parte de sus familiares, se encuentra rodeada de factores tales como; tristeza, aislamiento, dificultades para encontrarle un nuevo ánimo a sus vidas, ánimo para continuar viviendo, desolación, angustia e incluso ansiedad, y sentimiento profundo de culpa y angustia (Fuentes \& Flores, 2016). Adicional a otras afectaciones psicológicas que puedan surgir.

Estos conceptos nos obligan a dirigir vínculos de mediación (Gerónimo et al., 2021) como medidas tomadas por el estado a través de sus instituciones, como acciones positivas y necesarias para asegurar que las personas adultas mayores en situación de asilo en un marco de vida digna que revista el derecho a la salud desde su definición, es decir; desde el bienestar físico, emocional psicológico y espiritual, así desde una lógica de interdependencia y holística a como hemos referido anteriormente.

\section{Mediación y circulo restaurativo en procesos de convivencia del adulto mayor.}

Por lo que, la mediación y círculos restaurativos cuyo enfoque no solamente se limita a acercar a las partes a una pronta solución, donde se parte de un esquema netamente jurídico con intereses de las partes protegidos a partir de la presentación de un caso concreto y se evite por antonomasia un proceso judicial con todo y sus reglas de juego, este vínculo de mediación también nos dirige hacia un resultado holístico que procure un cambio transcendental, que impacte de manera positiva y se evite que la persona en este caso el adulto mayor sea víctima de contextos de vulnerabilidad genéricos de una situación de abandono, así mismo se atienda el resguardo de derechos como semillas que nacen y se reproduzcan valores familiares e institucionales para el bienestar.

Sin embargo, para que lo anterior se torne de carácter operativo es necesaria la inserción de una ley en el Estado sobre los derechos de las personas adultas mayores y derechos recíprocos entre sus familiares en el Estado, que refuerce la legalidad que parte del sistema de protección de salud dirigida a una vida que fortaleza los lazos de unión entre las personas adultas mayores que tienen la necesidad de encontrarse en un asilo o casa de retiro y sus familiares, partiendo del enfoque del ejercicio y reconocimiento de los derechos humanos.

Donde en esta ley parta de un sentido de vida digna, con enfoque policéntrico, donde se prevean espacios de convivencia dentro y fuera del asilo o casa de retiro, donde se encuentre de manera obligatoria, rescatando en todo momento la figura del vínculo familiar y responsabilidad de dignificar al adulto mayor desde el núcleo familiar y la promoción de valores.

Para logra lo anterior, resulta de manera esencial y como un requisito sine qua non para asegurar el ejercicio de estos derechos; es la implementación de una persona especializada en mediación y círculos restaurativos (Patiño \& Ruíz, 2015), con fusiones específicas y mediáticas que sirvan de puente entre las personas adultas mayores y sus familiares, bajo un contenido de goce de derechos recíprocos 
para este caso procurar la relación psico-afectivas entre padres e hijos, partiendo de un programa de vida digna, sugiriendo por parte del mediador la colaboración entre instituciones del estado para que en trabajo en conjunto, refuerce los vínculos de atención familiar que den como resultado una mayor eficacia al mediador adscrito al asilo.

Estos círculos restaurativos tendrán la finalidad de ser un método efectivo que permita la sana relación y el fortalecimiento de los lazos afectivos que a su vez dará certeza y fortaleza emocional que requieren este grupo que hoy es materia de estudio y control dentro de los asilos o casa de retiros, los cuales velaran por la integridad mental de la persona en este lugar hospedada.

Esta persona además de ser un especialista (Hernández et al., 2018) en derecho, psicología, sociología, debe desarrollar un sentido armónico de relación con los familiares de la persona, fungir como vínculo entre ellos, como de tener sus datos de localización y un estudio de campo donde ser palpe la realidad de ambas partes, procurando un marco de dialogo, operativo y afectivo entre y ellos y sus familiares en una situación de asilo e internado y muy especialmente sobre su relación con la persona.

Asegurarse que los distintos órganos involucrados, así como el personal de recursos humanos, se tengan con los suficientes recursos económicos, logísticos y científicos, para asegurar en un plano de inmediatez la protección del derecho de relación de los familiares con el adulto en situación de asilo.

\section{DISCUSIÓN}

La situación de estado de vulnerabilidad del adulto mayor puede ser nulificada a través de los procedimientos denominados círculos restaurativos. Los cuales brindan espacios de oportunidad para sanar heridas emocionales, entre la persona adulta mayor y sus familiares, quienes por diversas circunstancias han dejado de frecuentarlos una vez ingresados en estas casas de retiro, lo que eminentemente impactará de manera positiva en la condición psicoemocional de estas personas que caben dentro del bosquejo de la tercera edad. Procurando con ello la garantía del derecho a una vida digna, a salud, al disfrute familiar, entre otros desde la perspectiva de protección en su vulnerabilidad y no desde un boceto de obligación por parte de sus familiares.

\section{REFERENCIAS}

1. Corte IDH. (1999). Caso Villagrán Morales y otros vs Guatemala "Niños de la Calle", fondo, reparaciones y costas, Serie C, No. 63, párr. 144. Costa Rica.

2. Directorio Estadístico Nacional de Unidades Económicas (DENUE) del Instituto Nacional de Estadística y Geografía (INEGI). (2018). Adultos mayores en México.

3. Fuentes Reyes, Gabriela, \& Flores Castillo, Fernando Daniel. (2016). La indigencia de adultos mayores como consecuencia del abandono en el Estado de México. Papeles de población, 22(87), 161-181.

4. Gerónimo González, Paulino, Silva Hernández, Francisca \& Martínez Prats, Germán. (2021). Mecanismos alternativos en la solución de conflictos para la construcción de una cultura de paz. Ciencias de la Documentación, 7(1), 15-23.

5. Hernández Aguirre, Christian Norberto, Mendivil Torres, Jessica \& Hernández Aguirre, Cynrhia Vedelí. (2018). Los métodos alternativos de solución de controversias y sus facilitadores en el sistema penal acusatorio mexicano: hacia una justicia restaurativa, reparación del daño Integral y reinserción social. Ciencia Jurídica, 7(14), 13-30.

6. OMS. (1946). Preámbulo de la Constitución de la Organización Mundial de la Salud, adoptada por la Conferencia Sanitaria Internacional. Nueva York. 
7. Parra Vera, Oscar. (2013). Justiciabilidad de los derechos, económicos, sociales y culturales ante el sistema interamericano. CNDH, México.

8. Patiño Mariaca, Daniel Mauricio \& Ruíz Gutiérrez, Adriana María. (2015). La justicia restaurativa: un modelo comunitarista de resolución de conflictos. REVISTA DE LA FACULTAD DE DERECHO Y CIENCIAS POLÍTICAS - UPB, 45(122), 213-255.

9. Rodríguez R. (2000). Geriatría. Madrid: McGrawHill. 